
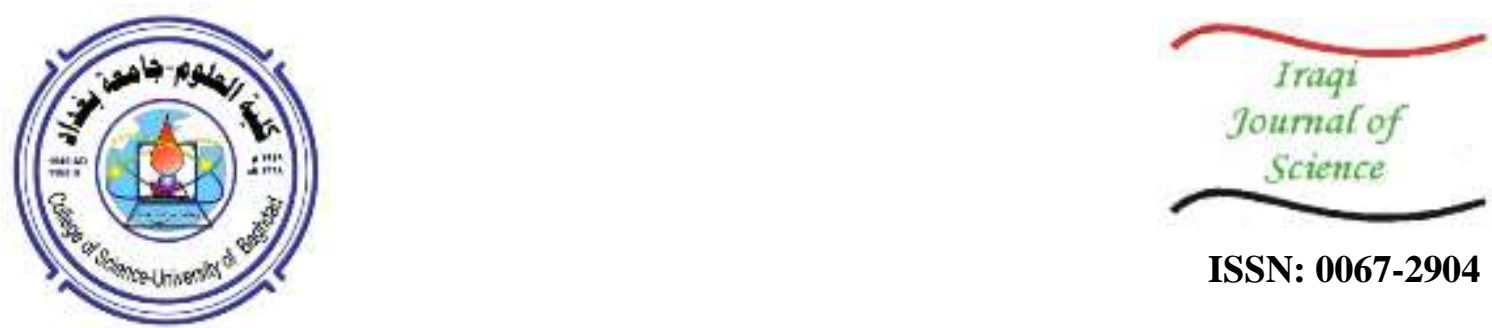

ISSN: 0067-2904

\title{
Bit Plane Slicing, Wavelet and Polynomials Mixing for Image Compression Rana Talib Al-Timimi
}

Department Banking and Financial Sciences, College of Management and Economic, Al-Mustansiriyah University, Iraq

\author{
Received: 28/4/2019 Accepted: 17/ 7/2019
}

\begin{abstract}
This paper introduced a hybrid technique for lossless image compression of natural and medical images; it is based on integrating the bit plane slicing and Wavelet transform along with a mixed polynomial of linear and non linear base. The experiments showed high compression performance with fully grunted reconstruction.
\end{abstract}

Keywords: Bit Plane Slicing, Wavelet Transform, Lossless Image Compression, Polynomial Prediction.
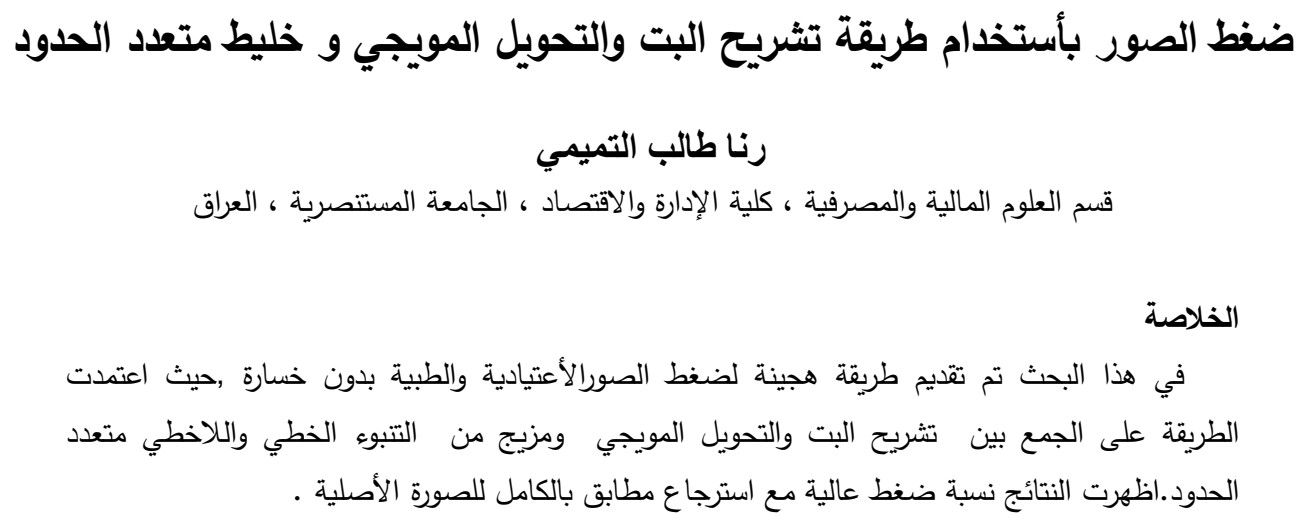

Introduction

Lossless compression of images is characterized by maintaining the quality of an image and ,therefor, the reconstructed image is the same as the original, but the compression ratio has a small values recorded as less than 10 [1]. This is because of focusing on the coding redundancy and/or inter pixel redundancy.

Researchers in recent years exploit ways to increase the lossless compression system's efficiency either by designing a technique that selects certain blocks and ignores the others or by merging several techniques such as the prediction and wavelet [2-6].

By applying the wavelet transform, the best compression ratio will be attained as compared to the spatial domain [7] where the image is decomposed into four sub bands (LL, HL, LH, and HH sub bands images). This process may be applied several times on the LL sub band image. The approximation sub band (LL) contains all image information; thus, it is considered as the most important part, while the other sub-bands are considered less significant as they contain very little amount of image information [8].

Bit Plane Slicing (BPS) was adopted as a technique combined with the well-known image compression methods by several researchers [9-11]; it is a separation technique used to generate eight binary images (eight layers: layer0 - layer7) according to bit position, where Low Order Layers (LOLs) represented by layers0-layer3 have small significant image details and therefore is ignored, while the High Order Layers (HOLs) (layer4-layer7) contain the most significant image details [12].

*Email: rana_talib@uomustansiriyah.edu.iq 
This paper introduces a technique to compress gray images based on determining the wavelet transform, along with a mixed polynomial of first and second order representation, that effectively improved the compression ratio.

\section{Materials and Methods}

The main concerns taken in the suggested method are:

Keeping the high order layers and neglecting the low order layers of bit plane slicing effectively reduces the number of bits from 8 to 4 bits, which means an implicit reduction in image information. Integrating the Wavelet Transform with the mixed polynomial coding of linear and non linear approximation model was achieved to compress the image. Figure-1 clearly illustrates the layout of the proposed method.

The proposed hybrid compression system implementation is described in details below.

1-The input: gray scale image (I) of size $n * n$.

2- Bit-Plane Slicing (BPS) was used to was used to produce eight binary bit plane images form image I, each plane was of size $n * n$. The higher top order bit planes (4-7) of significant major image were used and the lower order bit planes (0-3) were discarded.

3- The wavelet transform was exploited to produce four quadrants sub bands (LL and detail sub bands LH, HL and HH) each of size $n / 2 \times n / 2$; then we applied the polynomial approximation model on the LL sub-band, such that:

For bit slicing 7 non-linear polynomial prediction was applied on approximation of the sub band LL7 Otherwise for bit planes 4,5,6 linear polynomial prediction was used on approximation of the sub bands LL4.LL5 and LL6.

4- for bit slicing 7:

the approximated Image $L \widetilde{L} 7$ was determined using equation (15).

the error (residual) was found using equation (16).

5- for bits slicing 4,5,6:

the approximated Images $L \widetilde{L} 4, L \widetilde{L} 5$ and $L \widetilde{L} 6$ were determined using equation (20).

the errors (residual) $L L 4 \operatorname{Re} s d, L L 5 \operatorname{Re} s d$ and $L L 6 \operatorname{Re} s d$ were found using equation (21).

non-linear polynomial prediction $[13,14]$ was applied using the following equations:

$$
\begin{aligned}
& a_{1}-\frac{\sum_{i=0}^{n-1 / 2} \sum_{j=0}^{n-1 / 2}}{\sum_{i=0}^{n-1 / 2} \sum_{j=0}^{n}-1 / 2} \frac{j) \times\left(j-x_{c}\right)}{\left(j-x_{c}\right)^{2}} \\
& a_{2}=\frac{\sum_{i=0}^{n-1 / 2} \sum_{j=0}^{n-1 / 2} L L 7(i, j) \times\left(i-y_{c}\right)}{\sum_{i=0}^{n-1 / 2} \sum_{j=0}^{n-1 / 2}\left(i-y_{c}\right)^{2}} \\
& a_{5}=\frac{\sum_{i=0}^{n-1 / 2} \sum_{j=0}^{n-1 / 2 n} L / 27(i, j)(j-x c)(i-y c)}{\sum_{i=0}^{n} \sum_{j=0}^{n-1 / 2}(j-x c)^{2}(i-y c)^{2}} \\
& x c \quad c-\frac{n-1}{2} \\
& \mathrm{n} / 2 \times \mathrm{n} / 2 \\
& \left.W_{2}=\sum_{j=v}^{n-j} \quad x c\right)^{2}=\sum_{i=0}^{n-1 / 2}(i-y c)^{2}
\end{aligned}
$$




$$
\begin{aligned}
& \mathrm{W}_{3}=\sum_{\mathrm{j}=0}^{\mathrm{n}-1 / \mathrm{t}} \quad \mathrm{I}^{4}=\sum_{\mathrm{i}=0}^{\mathrm{n}-1 / 2}(\mathrm{i}-\mathrm{yc})^{4} \\
& \mathrm{~W}_{4}=\sum_{\mathrm{i}=\mathrm{v}}^{\mathrm{n} \cdot \cdots} \sum_{\mathrm{j}=0}^{1 / 2}(\mathrm{j}-\mathrm{xc})^{2}(\mathrm{i}-\mathrm{yc})^{2} \\
& \mathrm{~V}_{1}=\mathrm{a}_{0} \mathrm{~W}_{1}+\mathrm{a}_{3} \mathrm{~W}_{2}+\mathrm{a}_{4} \mathrm{~W}_{2} \\
& v_{2}-a_{0} W_{2}+a_{3} W_{3}+a_{4} w_{4} \\
& \mathrm{~V}_{3}=\mathrm{a}_{0} \mathrm{~W}_{2}+\mathrm{a}_{3} \mathrm{w}_{4}+\mathrm{a}_{4} \mathrm{~W}_{3}
\end{aligned}
$$

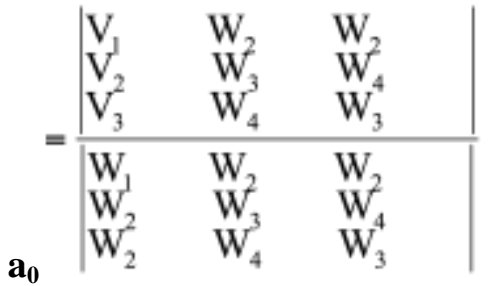

$$
=\frac{\left|\begin{array}{lll}
\mathrm{W}_{1} & \mathrm{~V}_{4} & \mathrm{~W}_{2} \\
\mathrm{~W}_{2} & \mathrm{~V}_{2} & \mathrm{~W}_{4} \\
\mathrm{~W}_{2} & \mathrm{~V}_{3} & \mathrm{~W}_{3}
\end{array}\right|}{\left|\begin{array}{lll}
\mathrm{W}_{1} & \mathrm{~W}_{2} & \mathrm{~W}_{2} \\
\mathrm{~W}_{2} & \mathrm{~W}_{3} & \mathrm{~W}_{4} \\
\mathrm{~W}_{2} & \mathrm{~W}_{4} & \mathrm{~W}_{3}
\end{array}\right|}
$$

$$
=\frac{\left|\begin{array}{lll}
\mathrm{W}_{1} & \mathrm{~W}_{2} & \mathrm{~V}_{1} \\
\mathrm{~W}_{2} & \mathrm{~W}_{3} & \mathrm{~V}_{2} \\
\mathrm{~W}_{2} & \mathrm{~W}_{4} & \mathrm{~V}_{3}
\end{array}\right|}{\left|\begin{array}{lll}
\mathrm{W}_{4} & \mathrm{~W}_{2} & \mathrm{~W}_{2} \\
\mathrm{~W}_{2} & \mathrm{~W}_{3} & \mathrm{~W}_{4}^{2} \\
\mathrm{~W}_{2} & \mathrm{~W}_{4} & \mathrm{~W}_{3}
\end{array}\right|}
$$

$$
L \tilde{L} 7=a 0 W 1+a 1(j-x c)+a 2(i-y c)+a 3(j-x c)^{2}+a 4(i-y c)^{2}+a 5(j-x c) \cdot(i-y c)
$$




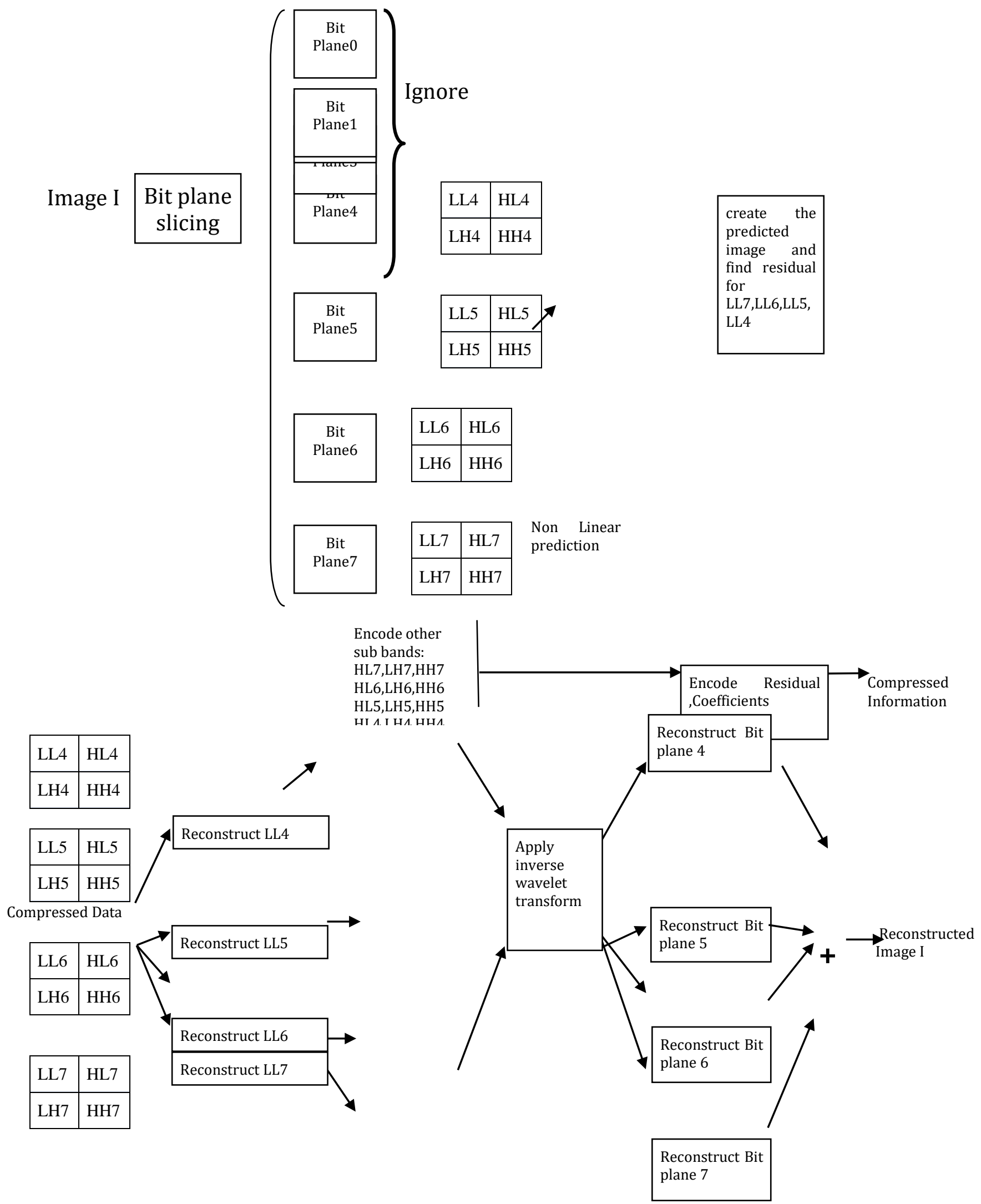

Figure 1-Compression and decompression System Structure

$$
\text { LL7 Resd = L7 - L L̃7 }
$$


The linear prediction coefficients were adopted [14,15] using the following equations :

$$
\begin{aligned}
& \mathrm{a}_{0}=\frac{1}{\mathrm{n} / 2 \times \mathrm{n} / 2} \sum_{\mathrm{i}=0}^{\mathrm{n}-1 / 2} \sum_{\mathrm{j}=0}^{\mathrm{n}-1 / 2} \mathrm{LL}(\mathrm{i}, \mathrm{j}) \\
& a_{1}=\frac{\sum_{i=0}^{n-1 / 2} \sum_{j=0}^{n-1 / 2} L L(i, j) \times\left(j-x_{c}\right)}{\sum_{i=0}^{n-1 / 2} \sum_{j=0}^{n-1 / 2}\left(j-x_{c}\right)^{2}} \\
& a_{2}=\frac{\sum_{i=0}^{n-1 / 2} \sum_{j=0}^{n-1 / 2} L L(i, j) \times\left(i-y_{c}\right)}{\sum_{i=0}^{n-1 / 2} \sum_{j=0}^{n-1 / 2}\left(i-y_{c}\right)^{2}} \\
& \mathrm{~L} \tilde{\mathrm{L}}=\mathrm{a} 0+\mathrm{a} 1\left(\mathrm{j}-\mathrm{x}_{\mathrm{c}}\right)+\mathrm{a} 2\left(\mathrm{i}-\mathrm{y}_{\mathrm{c}}\right)
\end{aligned}
$$

6-The residual $[15,16,17]$ was calculated as follows:

$$
L L \operatorname{Re} s d=L-L \widetilde{L}
$$

7-To reconstruct the original image:

A- LL quadrant 4,5,6 \& 7 were reconstructed by adding the residual to the predicted data:

$$
L L=L \widetilde{L}+L L \operatorname{Re} s d
$$

B-the bit plane was reconstructed by using the inverse wavelet transforms.

\section{Results and Discussion}

As a lossless compression, the performance measures were based on determining the Compression Ratio (CR) as in equation (23).

Compression Ratio $(\mathrm{CR})=\quad$ Size of Original Image

\section{Size of Compressed Information}

Two sets of images (natural and medical) were tested to report the performance of the proposed hybrid system (Figure-2). All tested images were of 256 gray levels (8bits/pixel) and of the size $256 \times 256$. Figure-3 illustrates the output images after the decompression process, while Figure 4 shows the eight layers, bit planes slicing for the tested input images.

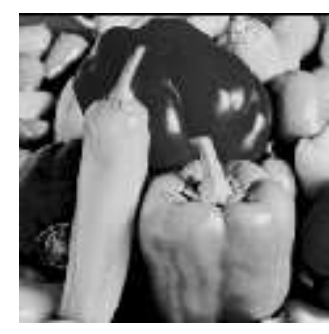

$\mathbf{a}$

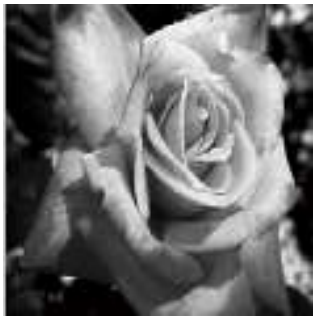

B

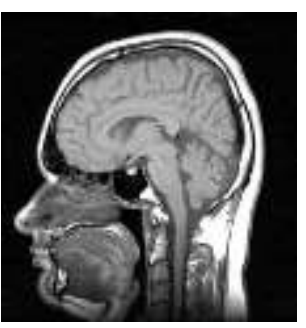

c

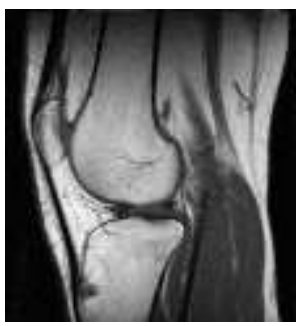

d

Figure 2-The Tested Grayscale Images, (a) Pepper (b) Rose (c) Brain and (d) Knee

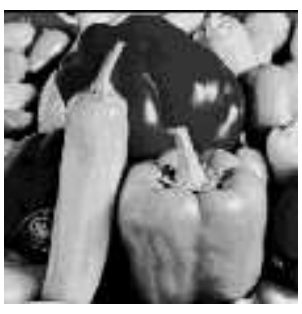

$\mathbf{a}$

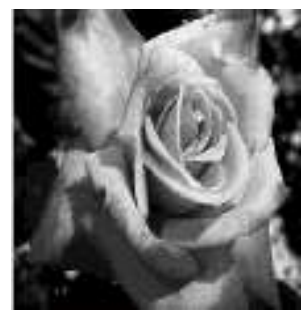

b

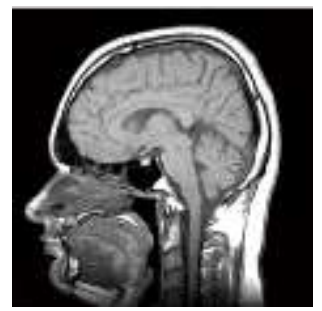

c

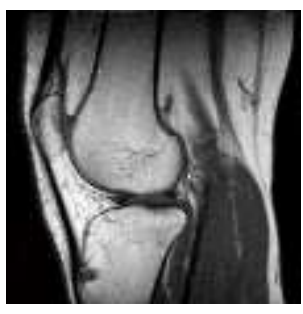

d 
Figure 3-The Output Images after the Decompression(a) Pepper (b) Rose (c) Brain and (d) Knee
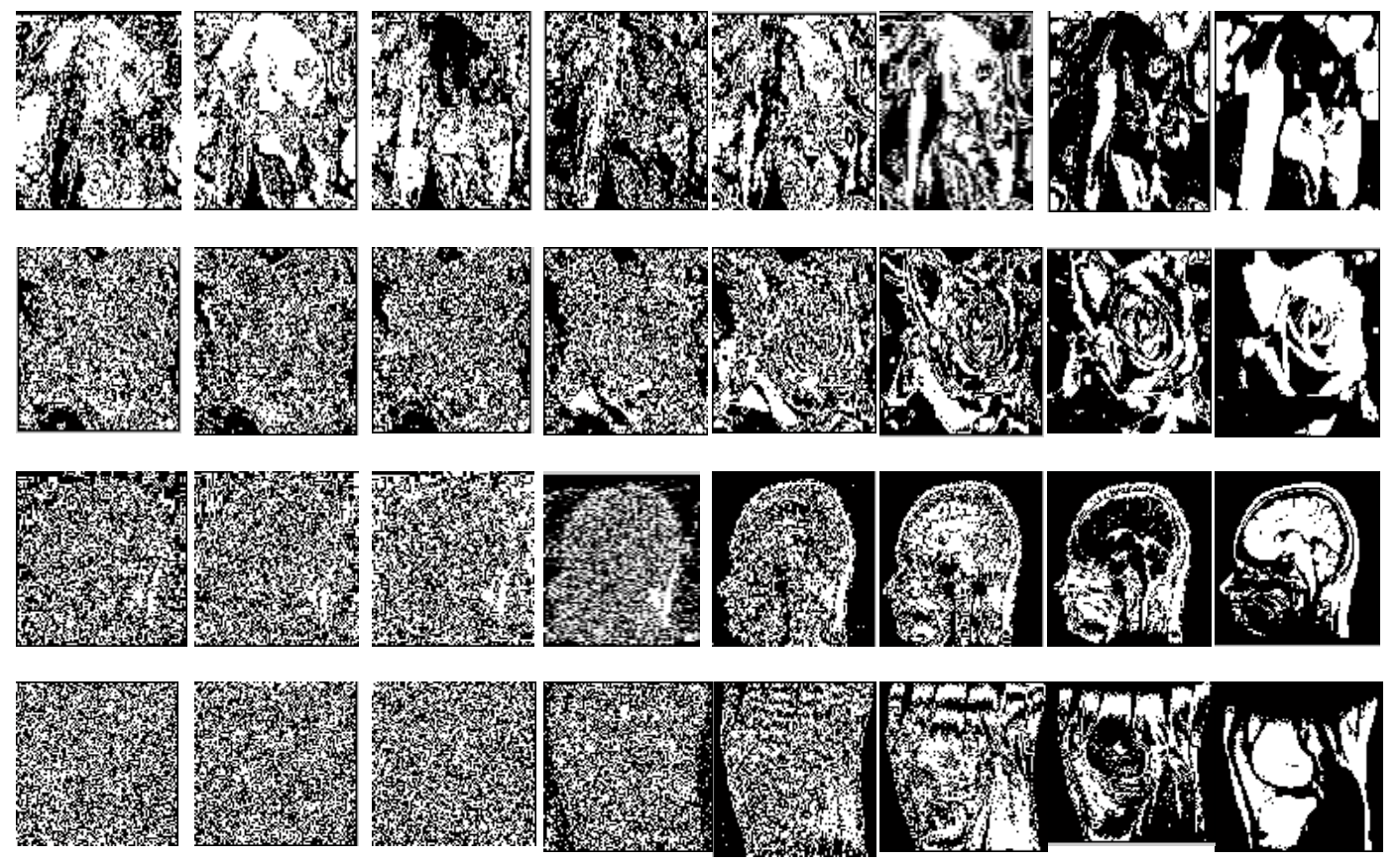

Figure 4-Bit plane slicing of Tested Images from Layer 0 to Layer 7.

Table-1 illustrates the compression performance of the suggested hybrid compression technique using two blocks sizes $(4 * 4 \& 8 * 8)$.

Tables-(2 and 3$)$ show the compression performance of two compression techniques, the traditional (nonlinear and linear, respectively).

The suggested hybrid lossless system was fast and simple. It was also obvious from the test results reported in Table-1 that the compression ratio was improved as compared with the results of the other methods (listed in Tables-(2 and 3).

Table 1-Performance of the Suggested Technique

\begin{tabular}{|c|c|c|c|c|c|}
\hline \multicolumn{2}{|c|}{} & \multicolumn{2}{|c|}{ Block size 4*4 } & \multicolumn{2}{c|}{ Block size 8*8 } \\
\hline $\begin{array}{c}\text { Test } \\
\text { image }\end{array}$ & $\begin{array}{c}\text { original } \\
\text { image size } \\
\text { (in bytes) }\end{array}$ & $\begin{array}{c}\text { compressed } \\
\text { information } \\
\text { size (in bytes) }\end{array}$ & $\begin{array}{c}\text { Compression } \\
\text { Ratio }\end{array}$ & $\begin{array}{c}\text { compressed } \\
\text { information size } \\
\text { (in bytes) }\end{array}$ & $\begin{array}{c}\text { Compression } \\
\text { Ratio }\end{array}$ \\
\hline Pepper & 65536 & 5878 & 11.1494 & 5462 & 11.9985 \\
\hline Rose & 65536 & 5624 & 11.6529 & 5240 & 12.5069 \\
\hline Brain & 65536 & 5610 & 11.6820 & 5208 & 12.5837 \\
\hline knee & 65536 & 6824 & 9.6038 & 5608 & 11.6862 \\
\hline
\end{tabular}


Table 2-Performance of non linear Traditional Compression System

\begin{tabular}{|c|c|c|c|c|c|}
\hline \multicolumn{2}{|c|}{} & \multicolumn{2}{|c|}{ Block size 4*4 } & \multicolumn{2}{c|}{ Block size 8*8 } \\
\hline $\begin{array}{c}\text { Test } \\
\text { image }\end{array}$ & $\begin{array}{c}\text { original } \\
\text { image size } \\
\text { (in bytes) }\end{array}$ & $\begin{array}{c}\text { compressed } \\
\text { information } \\
\text { size (in bytes) }\end{array}$ & $\begin{array}{c}\text { Compression } \\
\text { Ratio }\end{array}$ & $\begin{array}{c}\text { compressed } \\
\text { information size } \\
\text { (in bytes) }\end{array}$ & $\begin{array}{c}\text { Compression } \\
\text { Ratio }\end{array}$ \\
\hline Pepper & 65536 & 7738 & 8.4694 & 7110 & 9.2174 \\
\hline Rose & 65536 & 7100 & 9.2304 & 5968 & 10.9812 \\
\hline Brain & 65536 & 7324 & 8.9481 & 7310 & 8.9653 \\
\hline knee & 65536 & 7234 & 9.0594 & 6320 & 10.3696 \\
\hline
\end{tabular}

Table 3-Performance of Linear Traditional Compression System

\begin{tabular}{|c|c|c|c|c|c|}
\hline \multicolumn{2}{|c|}{} & \multicolumn{2}{|c|}{ Block size 4*4 } & \multicolumn{2}{c|}{ Block size 8*8 } \\
\hline $\begin{array}{c}\text { Test } \\
\text { image }\end{array}$ & $\begin{array}{c}\text { image size } \\
\text { (in bytes) }\end{array}$ & $\begin{array}{c}\text { compressed } \\
\text { information } \\
\text { size (in bytes) }\end{array}$ & $\begin{array}{c}\text { Compression } \\
\text { Ratio } \\
\text { compressed } \\
\text { information size } \\
\text { (in bytes) }\end{array}$ & $\begin{array}{c}\text { Compression } \\
\text { Ratio }\end{array}$ \\
\hline Pepper & 65536 & 8018 & 8.1736 & 77730 & 8.4781 \\
\hline Rose & 65536 & 7312 & 8.9628 & 6434 & 10.1859 \\
\hline Brain & 65536 & 8528 & 7.6848 & 8024 & 8.1675 \\
\hline knee & 65536 & 6952 & 9.4269 & 6436 & 10.1827 \\
\hline
\end{tabular}

\section{Conclusions}

The suggested hybrid technique is characterized by simplicity and efficiency. Combining the bit plane slicing with wavelet transforms leads to saving more storage space and hence affects the compression ratio due to preserving layers (4-7) and ignoring layers (0-3) from the bit plane, ultimately reducing the number of bits from 8 to 4 which implicitly means a reduction in image information. Finally, the compression ratio depended on the approximation of sub band LL block size, such that when the size gets bigger, fewer coefficients are needed, and this will implicitly improves the compression ratio

\section{References}

1. Francesc, A., Pep, M. and Vicent, R. 2013. Lossless and near-lossless image compression based on multiresolution analysis. Journal of Computational and Applied Mathematics, 242: 81-70.

Available at: $\quad$ https://www.sciencedirect.com/science/article/pii/S0377042712004682 doi:10.1016/j.cam.2012.10.028

2. Rana, Al-T. 2017. Hybrid Lossless Image Compression Using Wavelet Transform and Hierarchical non Linear Prediction. Al-Mustansiriyah Journal of Science, 28(2): 155-150

Available at: http://mjs.uomustansiriyah.edu.iq/ojs1/index.php/MJS/article/view/510 doi: http://dx.doi.org/10.23851/mjs.v28i2.510 
3. Ghadah, Al-K. ,Haider, Al-M. 2013. Lossless Compression of Medical Images using Multiresolution Polynomial Approximation Model. International Journal of Computer Applications, 76(3): 42-38.

Available at:

https://www.researchgate.net/publication/260845363_Lossless_Compression_of_Medical_Images using Multiresolution Polynomial Approximation Model doi: $10.5120 / 13230-0659$

4. Ghadah, Al-K. 2014. Wavelet Transform and Polynomial Approximation Model for Lossless Medical Image Compression. International Journal of Advanced Research Computer Science and Software Engineering, 4(3): 587- 584.

Available at: https://pdfs.semanticscholar.org/80ef/94afde38f3c6fc0e49db210365a023b946b5.pdf

5. Ghadah, Al-K., George, L. E. 2013. Fast Lossless Compression of Medical Images based on Polynomial. International Journal of Computer Applications, 70(15): 32-28.

Available at:

https://www.researchgate.net/publication/269666825_Fast_Lossless_Compression_of_Medical_I mages_based_on_Polynomial

doi: $10.5120 / 12039-7999$

6. George, L. E. and Sultan, B. 2011. Image Compression Based on Wavelet, Polynomial and Quadtree. Journal of Applied Computer Science \& Mathematics, 11(5): 20-15.

Available at:

https://www.researchgate.net/publication/267713119 Image Compression_Based On Wavelet Polynomial and Quadtree

7. Anantha, S., Eswaran, P. and Senthil, C. 2016. Arab journal for Science and Engineering. 41(8): 3061-3070.

Available at: https://link.springer.com/article/10.1007/s13369-016-2082-x doi:10.1007/s13369-016-2082-x .

8. Faisel, G.M. 2006. Color Image Compression Based on DWT. Ph.D. Thesis. College of science, University of Baghdad ,Iraq.

9. Podlasov, A. and Franti, P. 2006. Lossless Image Compression via Bit-Plane Separation and Multilayer Context Tree Modeling. J. of Electronic Imaging, 15(4): 10-1.

Available at: https://www.spiedigitallibrary.org/journals/Journal-of-Electronic-Imaging/volume15/issue-4/043009/Lossless-image-compression-via-bit-plane-separation-and-multilayercontext/10.1117/1.2388255.short doi: $10.1117 / 1.2388255$.

10. Santanu, H., Debotosh, B., Mita, N. and Dipak, K . 2012. A Low Space Bit-Plane Slicing Based Image Storage Method using Extended JPEG Format. International Journal of Emerging Technology and Advanced Engineering. 2(4): 699-694.

Available at:

https://pdfs.semanticscholar.org/6722/3e6b8c675ae7c40227b92b0996d6e84ba10d.pdf

11. Varun, B. and Dalveer, K. 2012. Lossless Compression Technique Using LZW with DFRLC. International Journal of Computer Science. 9: 427-425.

Available at:

https://www.researchgate.net/publication/264888336_Lossless_Compression_Technique_Using_I LZW_with_DFRLC

12. Pasumpon, P. and Sivanandam, N. 2012. Hybrid Algorithm for Lossless Image Compression using Simple Selective Scan order with Bit Plane Slicing. Journal of Computer Science. 8: 13451338 .

Available at:

https://www.researchgate.net/publication/289170093 Hybrid Algorithm for Lossless Image Co mpression using Simple Selective Scan order with Bit Plane Slicing doi: 10.3844/jessp.2012.1338.1345.

13. Rasha, Al-T. 2015. Image compression using Enhancement Polynomial Prediction Coding. Master thesis, University of Baghdad, Iraq.

14. George, L.E. and Ghadah Al-K. 2015. Image Compression based on Non-Linear Polynomial Prediction Model. IJCSM, 4:97-91. 
Available at: https://www.ijcsmc.com/docs/papers/August2015/V4I8201510.pdf.

15. Rana, Al-T. 2016. Lossy Image Compression based on Differential Coding and Linear Polynomial. Journal of College of Education. 5: 442-433.

Available at: http://edumag.uomustansiriyah.edu.iq/index.php/edumag/article/view/523.

16. Haider, Al-M.and Zainab, Al-R. 2014. Lossless Image Compression based on Predictive Coding and Bit Plane Slicing. International Journal of Computer Applications, 93(1): 1-6.

Available at:

https://www.researchgate.net/publication/271157234 Lossless Image Compression based on P redictive_Coding_and_Bit_Plane_Slicing

doi: 10.5120/16176-2068.

17. Rana, Al-T. 2018. Lossy Images Compression Based on Multiresolution. Al-Mustansiriyah Journal of Science, 29(2): 155-150

Available at: http://mjs.uomustansiriyah.edu.iq/ojs1/index.php/MJS/article/view/242

doi: http://doi.org/10.23851/mjs.v29i2.242 